\title{
Probing Dark Energy via Weak Gravitational Lensing with the SuperNova Acceleration Probe (SNAP)
}

\author{
A White Paper to the Dark Energy Task Force
}

J. Albert ${ }^{1}$, G. Aldering 2 , S. Allam ${ }^{3}$, W. Althouse ${ }^{4}$, R. Amanullah ${ }^{5}$, J. Annis ${ }^{3}$, P. Astier ${ }^{6}$, M. Aumeunier ${ }^{7,8}$, S. Bailey ${ }^{2}$, C. Baltay ${ }^{9}$, E. Barrelet ${ }^{6}$, S. Basa ${ }^{8}$, C. Bebek ${ }^{2}$, L. Bergström ${ }^{5}$, G. Bernstein ${ }^{10}$, M. Bester ${ }^{11}$, B. Besuner ${ }^{11}$, B. Bigelow ${ }^{12}$, R. Blandford ${ }^{4}$, R. Bohlin ${ }^{12}$, A. Bonissent ${ }^{7}$, C. Bower ${ }^{13}$, M. Brown ${ }^{12}$, M. Campbell ${ }^{12}$, W. Carithers ${ }^{2}$, D. Cole ${ }^{14}$, E. Commins ${ }^{2}$, W. Craig ${ }^{4}$, T. Davis ${ }^{15,2}$, K. Dawson ${ }^{2}$, C. Day ${ }^{2}$, M. DeHarveng ${ }^{8}$, F. DeJongh ${ }^{3}$, S. Deustua ${ }^{16}$, H. Diehl ${ }^{3}$, T. Dobson ${ }^{11}$, S. Dodelson ${ }^{3}$, A. Ealet ${ }^{7,8}$, R. Ellis ${ }^{1}$, W. Emmet ${ }^{9}$, D. Figer ${ }^{12}$, D. Fouchez ${ }^{7}$, M. Frerking ${ }^{14}$, J. Frieman ${ }^{3}$, A. Fruchter ${ }^{12}$, D. Gerdes ${ }^{12}$, L. Gladney ${ }^{10}$, G. Goldhaber ${ }^{11}$, A.

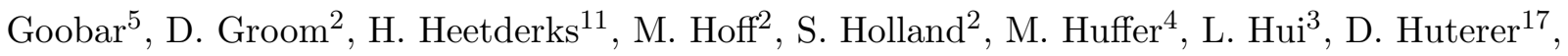
B. Jain ${ }^{10}$, P. Jelinsky ${ }^{11}$, C. Juramy ${ }^{6}$, A. Karcher ${ }^{2}$, S. Kent ${ }^{3}$, S. Kahn ${ }^{4}$, A. Kim ${ }^{2}$, W. Kolbe ${ }^{2}$, B. Krieger ${ }^{2}$, G. Kushner ${ }^{2}$, N. Kuznetsova ${ }^{2}$, R. Lafever ${ }^{2}$, J. Lamoureux ${ }^{2}$, M. Lampton ${ }^{11}$, O. Le Fèvre ${ }^{8}$, V. Lebrun ${ }^{8}$, M. Levi ${ }^{21}$, P. Limon ${ }^{3}$, H. Lin ${ }^{3}$, E. Linder ${ }^{2}$, S. Loken ${ }^{2}$, W. Lorenzon ${ }^{12}$, R. Malina ${ }^{8}$, L. Marian ${ }^{10}$, J. Marriner ${ }^{3}$, P. Marshall ${ }^{4}$, R. Massey ${ }^{18}$, A. Mazure ${ }^{8}$, B. McGinnis ${ }^{2}$, T. McKay ${ }^{12}$, S. McKee ${ }^{12}$, R. Miquel ${ }^{2}$, B. Mobasher ${ }^{12}$, N. Morgan ${ }^{9}$, E. Mörtsell ${ }^{5}$, N. Mostek ${ }^{13}$,

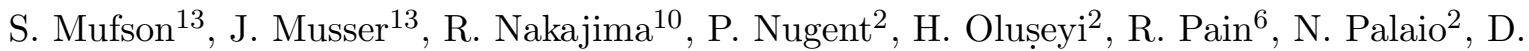
Pankow $^{11}$, J. Peoples ${ }^{3}$, S. Perlmutter ${ }^{22}$, D. Peterson ${ }^{2}$, E. Prieto ${ }^{8}$, D. Rabinowitz ${ }^{9}$, A. Refregier ${ }^{19}$, J. Rhodes ${ }^{1,14}$, N. Roe ${ }^{2}$, D. Rusin ${ }^{10}$, V. Scarpine ${ }^{3}$, M. Schubnell ${ }^{12}$, M. Seiffert ${ }^{14}$, M. Sholl ${ }^{11}$, H. Shukla ${ }^{11}$, G. Smadja ${ }^{20}$, R. M. Smith ${ }^{1}$, G. Smoot ${ }^{11}$, J. Snyder ${ }^{9}$, A. Spadafora ${ }^{2}$, F. Stabenau ${ }^{10}$, A. Stebbins ${ }^{3}$, C. Stoughton ${ }^{3}$, A. Szymkowiak ${ }^{9}$, G. Tarlé ${ }^{12}$, K. Taylor ${ }^{1}$, A. Tilquin ${ }^{7}$, A. Tomasch ${ }^{12}$, D. Tucker ${ }^{3}$, D. Vincent ${ }^{6}$, H. von der Lippe ${ }^{2}$, J-P. Walder ${ }^{2}$, G. Wang ${ }^{2}$, A. Weinstein ${ }^{1}$, W. Wester ${ }^{3}$ M. White ${ }^{11}$, saul@lbl.gov melevi@lbl.gov 


\section{Project Summary:}

SNAP is a candidate for the Joint Dark Energy Mission (JDEM) that seeks to place constraints on the dark energy using two distinct methods. The first, Type Ia SN, is discussed in a separate white paper. The second method is weak gravitational lensing, which relies on the coherent distortions in the shapes of background galaxies by foreground mass structures. The excellent spatial resolution and photometric accuracy afforded by a 2-meter space-based observatory are crucial for achieving the high surface density of resolved galaxies, the tight control of systematic errors in the telescope's Point Spread Function (PSF), and the exquisite redshift accuracy and depth required by this project. These are achieved by the elimination of atmospheric distortion and much of the thermal and gravity loads on the telescope. The SN and WL methods for probing dark energy are highly complementary and the error contours from the two methods are largely orthogonal.

The nominal SNAP weak lensing survey covers 1000 square degrees per year of operation in six optical and three near infrared filters (NIR) spanning the range $350 \mathrm{~nm}$ to $1.7 \mu \mathrm{m}$. This survey

\footnotetext{
${ }^{1}$ California Institute of Technology

${ }^{2}$ Lawrence Berkeley National Laboratory

${ }^{3}$ Fermi National Accelerator Laboratory

${ }^{4}$ Stanford Linear Accelerator Center

${ }^{5}$ University of Stockholm

${ }^{6}$ LPNHE, CNRS-IN2P3, Paris, France

${ }^{7}$ CPPM, CNRS-IN2P3, Marseille, France

${ }^{8}$ LAM, CNRS-INSU, Marseille, France

${ }^{9}$ Yale University

${ }^{10}$ University of Pennsylvania

${ }^{11}$ University of California at Berkeley

${ }^{12}$ University of Michigan

${ }^{12}$ Space Telescope Science Institute

${ }^{13}$ Indiana University

${ }^{14}$ Jet Propulsion Laboratory

${ }^{15}$ The Australian National University

${ }^{16}$ American Astronomical Society

${ }^{17}$ University of Chicago

${ }^{18}$ Cambridge University

${ }^{19}$ CEA, Saclay, France

${ }^{20}$ IPNL, CNRS-IN2P3, Villeurbanne, France

${ }^{1} \mathrm{Co}-\mathrm{PI}$

${ }^{2} \mathrm{PI}$
} 
will reach a depth of 26.6 AB magnitude in each of the nine filters and allow for approximately 100 resolved galaxies per square arcminute, $\sim 3$ times that available from the best ground-based surveys. Photometric redshifts will be measured with statistical accuracy that enables scientific applications for even the faint, high redshift end of the sample. Ongoing work aims to meet the requirements on systematics in galaxy shape measurement, photometric redshift biases, and theoretical predictions.

\section{Weak Lensing as a Probe of Dark Energy}

The study of modern cosmology has been tremendously advanced by probes for which detailed comparison of theory and observation is possible. Weak gravitational lensing (WL) is one such probe, combining theoretical control and experimental tractability with sensitivity to interesting cosmological parameters (see Refregier 2003 for a review). WL is the slight distortion in the images of distant source galaxies due to deflections of light rays by the inhomogeneous mass distribution of the Universe. The size of these distortions depends upon both $D(z)$, the distances traveled, and upon $G(z)$, the growth function which determines the amplitude of the deflecting mass concentrations. WL is an attractive cosmological probe because the physical effect, gravitational deflection of light, is simple and well understood. Furthermore, the deflecting masses are dominated by dark matter, the evolution of which is purely gravitational and hence calculable.

The sensitivity of WL to both distances and gravitational growth makes lensing a particularly powerful probe of dark energy, allowing us to differentiate models of dark energy from modifications to Einstein gravity (Linder 2005). WL power spectra and cross-spectra among 5-10 bins in redshift are the most robust measure of dark energy. Recent work has shown that the weak lensing sky contains information beyond the power spectrum: non-Gaussian signatures captured through the bispectrum (BS; 3-point shear correlations), highly non-linear features (galaxy clusters), and crosscorrelations with foreground galaxies. These three additional probes of dark energy with WL could be extremely powerful in conjunction, but their theory and measurement needs further investigation.

Because the weak lensing induced distortions on the shapes of individual galaxies are small $(\sim 0.1-2 \%)$ compared to the intrinsic scatter in the shapes of the galaxies $(\sim 30 \%)$, weak lensing must be measured statistically. A weak lensing measurement needs to overcome both this intrinsic scatter in galaxy shapes (by imaging a high surface density of resolved galaxies), and the noise on the measurement of each galaxy shape. This noise is predominantly due to the PSF (of the atmosphere, telescope, etc.). Lack of systematics control or a reduced number density of accurately resolved galaxies will limit the statistical impact of increased area.

To extract cosmological information from WL we need to:

- Measure the shapes and photometric redshifts (photo-z's) of very large numbers $\left(10^{8}\right.$ or more) of background galaxies on the sky.

- Remove the effect of telescope or atmospheric optics on the shapes of these galaxies.

- Develop statistics that extract cosmological information from a catalog of galaxy shapes and 
redshifts.

The advantage of space-based weak lensing dark energy measurements comes from the extent to which systematic errors in shape and redshift measurement can be controlled; ground-based observations have far larger and more variable optical distortions than would SNAP. Further, as shown below, poorer angular resolution makes ground-based surveys more susceptible to small errors in PSF treatment. Space provides important gains with the higher number density of galaxies, better photometric redshifts, and a broader redshift coverage which enables studies at smaller scales and higher redshift than possible from the ground.

\section{The SNAP Weak Lensing Survey}

The current baseline SNAP weak lensing survey calls for imaging 1000 square degrees over the course of roughly one year. With an extended mission this can be expanded by 1000 square degrees per year of operation. SNAP's unique focal plane design enables a step-and-stare strategy in which each of 6 optical and 3 near infrared filters covers the same patch of sky in a sequence (Albert et al. 2005). Four 300 second exposures would be taken with each optical filter (for cosmic ray rejection and better image sampling). The NIR filters would get twice the exposure time. Due to the high quantum efficiency of the LBL CCDs being developed for SNAP, this exposure time reaches a magnitude limit of about $26.6(\mathrm{AB})$ in each band for extended sources at a $\mathrm{S} / \mathrm{N}$ of 10 . The broad spectral coverage and high photometric accuracy will enable photometric redshifts for each galaxy with an accuracy $\Delta z<0.045$ out to magnitude 26 . The pixel scale of the SNAP CCDs $(0.1$ arcsecond per pixel) has been studied using both analytic methods and detailed image simulations which find that it is well suited for weak lensing studies with the tight SNAP PSF (Bernstein 2002; High et al. 2004; Figure 1).

A space-based observatory like SNAP will provide significant improvement in shape measurements. While the fundamental limitation of ground-based observing is largely due to atmospheric blurring (seeing) and sky noise, these do not enter for space-based measurements. Moreover, SNAP will also benefit from a more stable thermal environment and the absence of gravity loading, which will produce a stable PSF, and thus enable superior shape deconvolution (Rhodes et al. 2004). The noise due to intrinsic galaxy shapes will be minimized by resolving a high surface density of galaxies due to the small, stable PSF. Based on observations with the HST, we estimate SNAP will provide a threefold increase in the number of resolved galaxies compared to the best ground based surveys (about 100 galaxies per square arcminute; Massey et al 2004; Bernstein 2005; Figure 2). Equally important, the photometric stability and nine filter coverage of SNAP will facilitate accurate photo-z estimation out to $z \sim 3$.

Note that no space-based WL survey is likely to occur before SNAP; SNAP will represent a three order of magnitude improvement in this area over the current largest space-based WL survey, the COSMOS HST 2-square degree survey. 


\section{Systematic Errors}

Errors in measuring galaxy shapes can roughly be divided into multiplicative and additive errors (Huterer et al. 2005). The requirement for the SNAP mission is to control multiplicative error, likely due to errors in shear calibration and PSF size estimation, to about 0.01 or less (or about $1 \%$ of the mean shear in a redshift bin); see the right panel of Figure 3. Additive errors, which arise due to PSF anisotropy, require more detailed modeling, but a rough estimate shows that the contribution needs to be below $0.1 \%$ on scales of $\sim 10$ arcmin $(\ell \sim 1000)$ in order not to degrade the error in cosmological parameters. Furthermore, the redshift distribution of the source galaxies must be known with a bias $\sim 0.003$ per redshift bin or smaller. It should be noted that wider area surveys have stricter requirements on these systematics because the statistical errors are smaller. For a realistic assessment of the potential of SNAP and ground-based WL experiments, we must determine how well these systematic errors can be controlled, and estimate the number of galaxy shapes/redshifts that can be measured to sufficient accuracy in a given mission.

\subsection{Galaxy Shape Measurement}

To estimate the degree of anisotropy of the SNAP PSF, we use a ray tracer that incorporates all the optical elements of the SNAP telescope. Misalignments in the optics generate PSF anisotropy, which we characterize by ellipticity. The effect of three time varying effects: thermal drift, guider jitter, and structural vibration on the PSF have been estimated for expected parameters of the SNAP telescope. We generated multiple realizations of a thousand square degree mock survey to include the systematic error pattern induced by these effects. Their contribution to the power spectrum of the shear is shown in Figure 4. We find that the dominant effects come from guider jitter (which rises with increasing $\ell$ and thermal drift, which peaks at $\ell \sim 10^{3}$ ). The expected amplitude of the first effect is comparable to the statistical errors and would require correction. It is worth noting that ground based telescopes typically have PSF anisotropy at the percent level, which would lie above the signal in Figure 4 and require substantial corrections.

Residual PSF anisotropy can be reduced using measured stellar PSFs. Hence one must know (a) how many stellar images are available and how well they allow us to measure the systematics, and (b) how to correct galaxy images to the highest possible accuracy. Jarvis \& Jain (2004) have demonstrated a new method using principal component analysis (PCA) to analyze PSF data that efficiently recognizes PSF patterns that persist over multiple exposures. They show how this led to elimination of detectable systematic errors in existing ground-based data. We note that the SNAP PSF requires far smaller corrections than most ground based instruments; the PCA method is expected to effectively eliminate the additive PSF errors, since the correction improves with the number of exposures, which is very large for the SNAP survey.

Answering question (b) requires testing shear calibration. Shape-measurement algorithms based on the Bernstein \& Jarvis (2002) and Refregier \& Bacon (2003) approaches are being tested

using simulated data (since no real data can reach this accuracy yet) to push our accuracy to the sub-percent level that will be required. 
The Shear TEsting Program (STEP) is an ongoing effort by the weak lensing community to understand the systematic effects and biases inherent in current methods for measuring galaxy shapes and deconvolving those shapes from the telescope's PSF. Heymans et al (2005) presented the first analysis of the shear measurement methods of various groups on simulated galaxy images with known input shears. Subsequent tests on images with more realistic galaxy morphologies are underway. These tests will also include simulations of space-based images. Of particular importance to SNAP is the ability of these tests to point out areas in which current methods can be improved so that the required level of systematics can be achieved.

\subsection{Photometric Redshifts}

Accurate redshifts of source galaxies are necessary in order to determine the galaxies' overall distribution in redshift and also to subdivide them in several redshift bins for lensing tomography and cross-correlation analyses. Current photometric redshift techniques have redshift errors of several percent per galaxy, and this accuracy is improving as we are learning about the different techniques. It is estimated that the overall redshift bias (obtained by averaging over many galaxies in a redshift bin) needs to be at least an order of magnitude smaller (Huterer et al. 2005; see left panel of Figure 3) in order not to appreciably degrade the error in cosmological parameters. A similar requirement applies to the scatter in the bias per redshift bin (Ma et al. 2005). Work on understanding and improving the photometric techniques is ongoing, and the prospects are excellent as the requisite redshift requirements might already be achievable even with current techniques.

The broad spectral coverage and high photometric accuracy will enable photometric redshifts for each galaxy with an rms accuracy $\Delta z=0.045$ out to magnitude 26 . This is based on estimates using simulated galaxies and currently well-tested redshift estimation techniques. We note that if the number of filters is reduced to six optical filters, then for the same signal-to-noise the rms photo-z error increases to 0.065 , and has twice the outlier fraction; and for four optical filters it is 0.1 , with five times the outlier fraction (the outliers are not used in the error estimate; Dahlen et al. 2005, in preparation). The expected levels of bias need further study, as do the requirements of appropriate spectroscopic samples needed for calibration to reduce this bias to acceptable levels. This is an important task for precursor surveys and simulation studies.

\subsection{Theoretical Uncertainties}

For the non-linear power spectrum, we need to know the spectrum in the range $0.1<k<$ $10 \mathrm{Mpc}$ to $1-2 \%$ accuracy, with $k \sim 1$ being where the requirement is most stringent (Huterer $\&$ Takada 2005). The current state of the art on N-body simulations is $\sim 5 \%$ agreement on the relevant scales for dark matter simulations with some simulations agreeing better than that. There is no known obstacle to reducing the error to the SNAP requirements, provided only gravitational physics is included. For smaller scales the effects of baryonic processes are important, though even if uncorrected they only limit us to $\ell<3000$ (Zhan \& Knox 2005; White 2005). In the coming 
decade we can expect to be able to run thousands of realizations of particle mesh simulations with $\sim 1$ billion particles and box-lengths of about $500 \mathrm{Mpc}$, which would be sufficient for percent-level accuracy in the lensing power spectrum (Vale \& White 2003).

In order to extend the range of scales which we can use for constraints on dark energy we will pursue two complementary strategies. The first is to improve the modelling. There has been dramatic progress in modelling the major processes affecting the matter on sub-Mpc scales, and there is good reason to believe a small number of simulations including most of the relevant physics will be available before launch. Even phenomenological recipes can help. For example modelling the distribution of gas in hydrostatic equilibrium in a known potential will go a long way towards including the effect of hot gas in clusters while adiabatic contraction models will allow us to model the effect of the cold component. Even if these models are not precise, we should be able to reduce the residual effects by a factor of 2 or more.

The other route is to use the high quality of the SNAP observations to mitigate theory uncertainty. Since the latter is dominant on small scales this amounts to using photometric redshifts of the source galaxies to arrange that the lensing weight is peaked away from $z=0$ where the smallscale power has the most influence on any given angular scale. There exist several techniques for doing this (Huterer \& White 2005), the most promising being $k$-space cutting or simply eliminating source galaxies with low inferred redshifts.

With this knowledge, we would be able to use the small-scale resolution of SNAP to extract more cosmological information from the power spectrum and measure new statistics, such as higher order correlations. In our current forecasts, we have not relied on small scales that are affected by non-gravitational physics.

\subsection{Tests and Marginalization of Residual Systematics}

The magnitude and scale dependence of B-modes in the data is a useful monitor of systematics as they are not produced by scalar gravitational perturbation modes. There are numerous other tests, including the self-consistency of the power spectrum and bispectrum, that will be useful to check for systematic errors.

Weak gravitational lensing offers prospects for self-calibration (Huterer et al. 2005), where weak lensing data is used to concurrently determine both the systematic and the cosmological parameters. The effects of the parameterized systematics can then be marginalized out without the need to know their values (but at the expense of increasing the cosmological parameter errors). A particularly promising approach is to combine weak lensing methods (for example the PS and BS measurements) as in those cases the self-calibration may be achieved with a smaller degradation in cosmological parameter errors. Further study is needed to model systematics in greater detail and to allow for shape and redshift errors to be correlated. 


\subsection{Weak Lensing Measurements from Space}

One of the crucial advantages of a space-based mission will be control of systematic errors that is superior to that from ground-based surveys. Since the expected systematic errors from groundbased telescopes have yet to be precisely quantified, this advantage will become more evident as work on systematics progresses. Figure 5 shows a representation of how a space-based survey like SNAP is complementary to a much larger ground-based weak lensing survey such as LSST.

Figure 6 shows one way to estimate how the superior imaging of SNAP translates into improved systematic errors in shear measurements. There is at least an order of magnitude advantage in the resultant systematics. The ratio of the typical galaxy size to the PSF is thus critical not only for attaining a higher number density, but also to protect against systematic errors. In addition, a direct source of systematic errors is the anisotropy of the PSF. The stable thermal environment and absence of gravity loading will enable SNAP to have a far better controlled PSF than groundbased telescopes. Finally, the estimation of photo-z's is greatly aided by the superior photometric calibration and 9 filters planned for SNAP. Being able to image with all filters with the same instrument, and to span the NIR bands, will allow SNAP to attain an rms redshift error better than 0.05 out to $z=3$. This is critical, since without accurate redshift estimates, even accurate shear measurements could lead to biased estimates of cosmological parameters.

For given sky coverage the statistical errors are significantly better with SNAP due to the $\sim 3$ times higher number density of well-resolved galaxies. Thus, SNAP imaging will enable high redshift and small angular scale studies with a far greater precision than even the best possible ground-based survey. Given how little we know about dark energy, it is valuable to be able to probe a wide redshift range to be able to discover new effects in dark energy evolution.

\section{Relevance of Current and Ongoing Surveys}

Near term, ground based weak lensing surveys will cover hundreds (Canada-France-Hawaii Telescope Legacy Survey) to thousands (KiloDegree Survey, RCS2, DES, Pan-Starrs) of square degrees to moderate depth. These will mature the field of weak lensing for cosmological constraints. While the results will lack the precision and accuracy achievable with SNAP, they will provide large scale information and the opportunity to test galaxy shear and photo-z measurement methods. This

will lay the groundwork for the third generation experiment of SNAP, opening the window on the physics of the universe.

\section{Forecast Dark Energy Constraints from SNAP}

In addition to the strong leverage on cosmological parameter determination, and robustness in the use of two independent methods, the combination of WL and supernova distances enables a fundamental test of the framework: the theory of gravity. By combining distance information on the expansion history and WL information on the growth history, breakdowns or extensions of 
Einstein gravity can be distinguished from new physical components as the physics responsible for acceleration of the universe (Linder 2005). This is a critical advantage needed for true understanding of dark energy.

Table 1 shows the constraints on dark energy parameters with a 1000 square degree WL survey combined with the SN measurements. The parameter analysis is as described in the SN white paper. Note that significant gains in the WL constraints are expected on inclusion of bispectrum tomography (Takada \& Jain 2004) and cross-correlations between foreground galaxies and shear (Jain \& Taylor 2003; Bernstein \& Jain 2004; Song \& Knox 2004; Hu \& Jain 2004; Zhang et al, 2003). Further, a survey that lasts beyond the basic 1 year survey would improve the statistical errors which scale as $f_{\text {sky }}$. A 4000 square degree survey (3 additional years of survey time) would

lead to a factor of two improvement in the constraints from WL as well as being valuable for studies of baryon oscillations, galaxy clusters, and diverse astronomy.

\section{References}

Albert et al. 2005 PASP submitted, astro-ph/0405232

Bernstein 2005, in preparation

Bernstein \& Jain, 2004, ApJ, 600, 17

Bernstein \& Jarvis 2002, ApJ, 583, 123

Bernstein 2002, PASP, 114, 98

Dahlen et al, 2005, in preparation

Hamana et al, 2004, MNRAS 350, 893

Heymans et al, 2005, MNRAS submitted, astro-ph/0506112

High et al, 2004, BAAS, 205, 6502

Hoekstra, 2004, MNRAS, 347, 1337

$\mathrm{Hu} \&$ Jain, PhRvD, 70, 3009

Huterer et al, 2005, MNRAS submitted astro-ph/0506030

Huterer \& Takada, 2005, Astroparticle Physics, 23, 369

Huterer \& White 2005, PRD, submitted, astro-ph/0501451

Jain \& Taylor 2003, PRL, 91, 141302

Jarvis \& Jain, 2004, ApJ submitted, astro-ph/0412234

Linder, 2005 astro-ph/0507263

Ma, Hu \& Huterer, 2005, astro-ph/0506614 
Massey et al, 2004, AJ, 127, 3089

Refreiger \& Bacon, 2003, MNRAS, 338, 48

Refregier, 2003, ARA\&A, 41, 645

Rhodes et al, 2004, APh, 20, 377

Song \& Knox, 2004, PhRvD, 70, 3510

Takada \& Jain, 2004, MNRAS, 348, 897

Vale \& White, 2003, ApJ, 592, 699

White, 2005, APh, 23, 349

Zhan \& Knox, 2005, ApJ, 616, 75

Zhang et al, 2003, ApJ submitted, astro-ph/0312348 
Table 1. SNAP 1- $\sigma$ uncertainties in dark-energy parameters, with conservative systematics for the supernova and a 1000 sq. deg. weak-lensing survey. The WL survey does not include constraints from the BS or cross correlation.

\begin{tabular}{cccc}
\hline \hline Model & $\sigma_{\Omega_{w}}$ & $\sigma_{w_{0}}$ & $\sigma_{w^{\prime}}$ \\
\hline
\end{tabular}

Fiducial Universe: flat, $\Omega_{M}=0.3$, Cosmological Constant dark energy

SNAP SN + WL; flat; $w(z)=w_{0}+2 w^{\prime}(1-a) \quad 0.005 \quad 0.05 \quad 0.11$

Fiducial Universe: flat, $\Omega_{M}=0.3$, SUGRA-inspired dark energy

$$
\text { SNAP SN + WL; flat; } w(z)=w_{0}+2 w^{\prime}(1-a) \quad 0.005 \quad 0.03 \quad 0.06
$$

Note. - Cosmological and dark-energy parameter precisions for two fiducial flat universes with $\Omega_{M}=0.3$ : one in which the dark energy is attributed to a Cosmological Constant and the other to a SUGRA-inspired dark-energy model. The parameter precisions then depend on the choice of data set, priors from other experiments, assumptions on the flatness of the universe, and the model for the behavior of $w$. In this paper we define $w^{\prime} \equiv-d w /\left.d \ln a\right|_{z=1}$ 


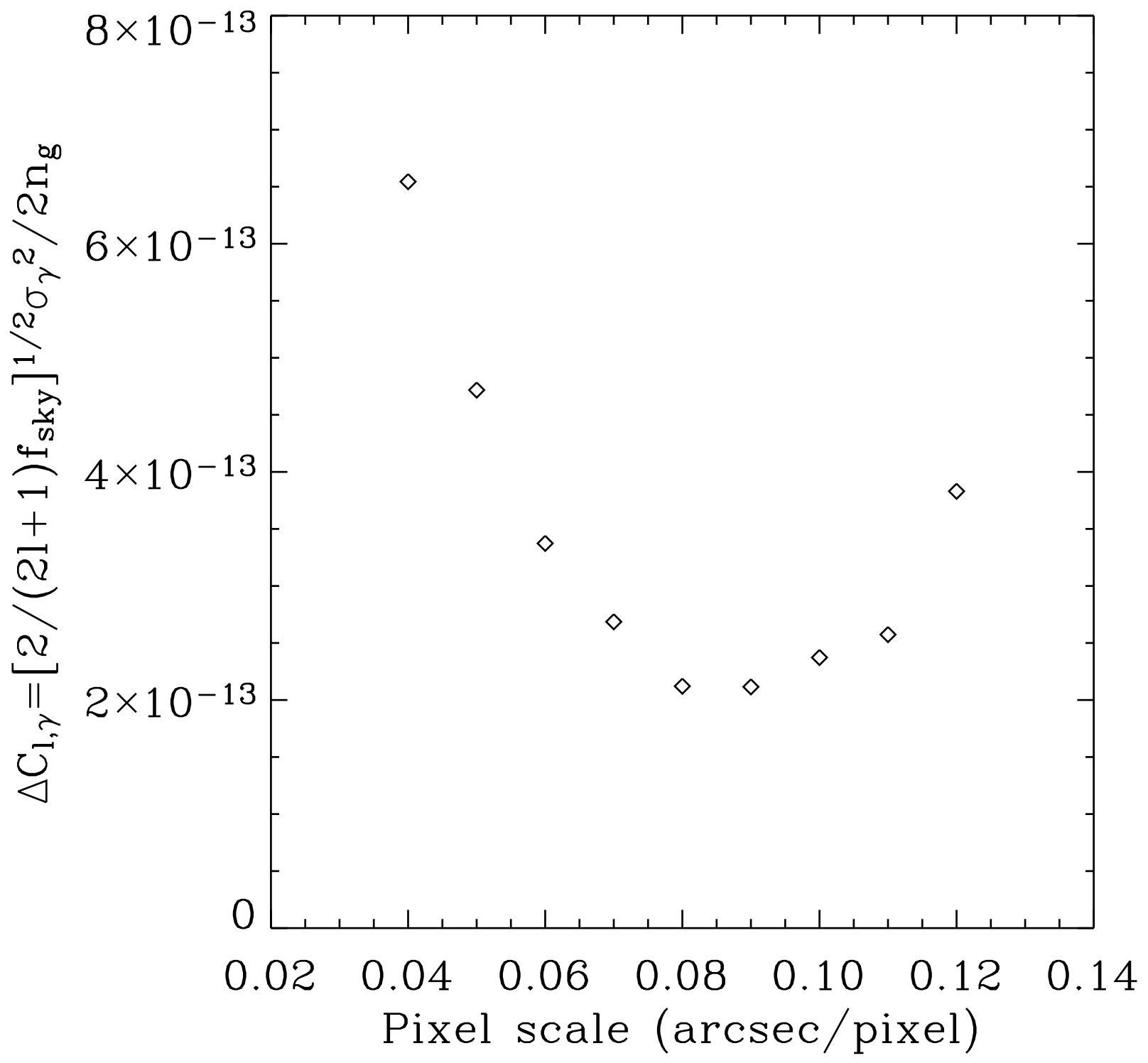

Fig. 1. - This plot shows the preliminary results of a study aimed at optimizing the pixel scale of the SNAP telescope for weak lensing. The y-axis of the plot shows the error $\Delta C_{l}$ on the matter power spectrum, for a fixed survey time and number of pixels. That is, for a smaller pixel scale, the total area surveyed is smaller. It shows that the weak lensing efficiency of SNAP is relatively flat for pixels scales near 0.1 arcseconds, the nominal SNAP pixel scale. These results are conservative in that they do not account for dithering, which would shift the optimum to the right. 


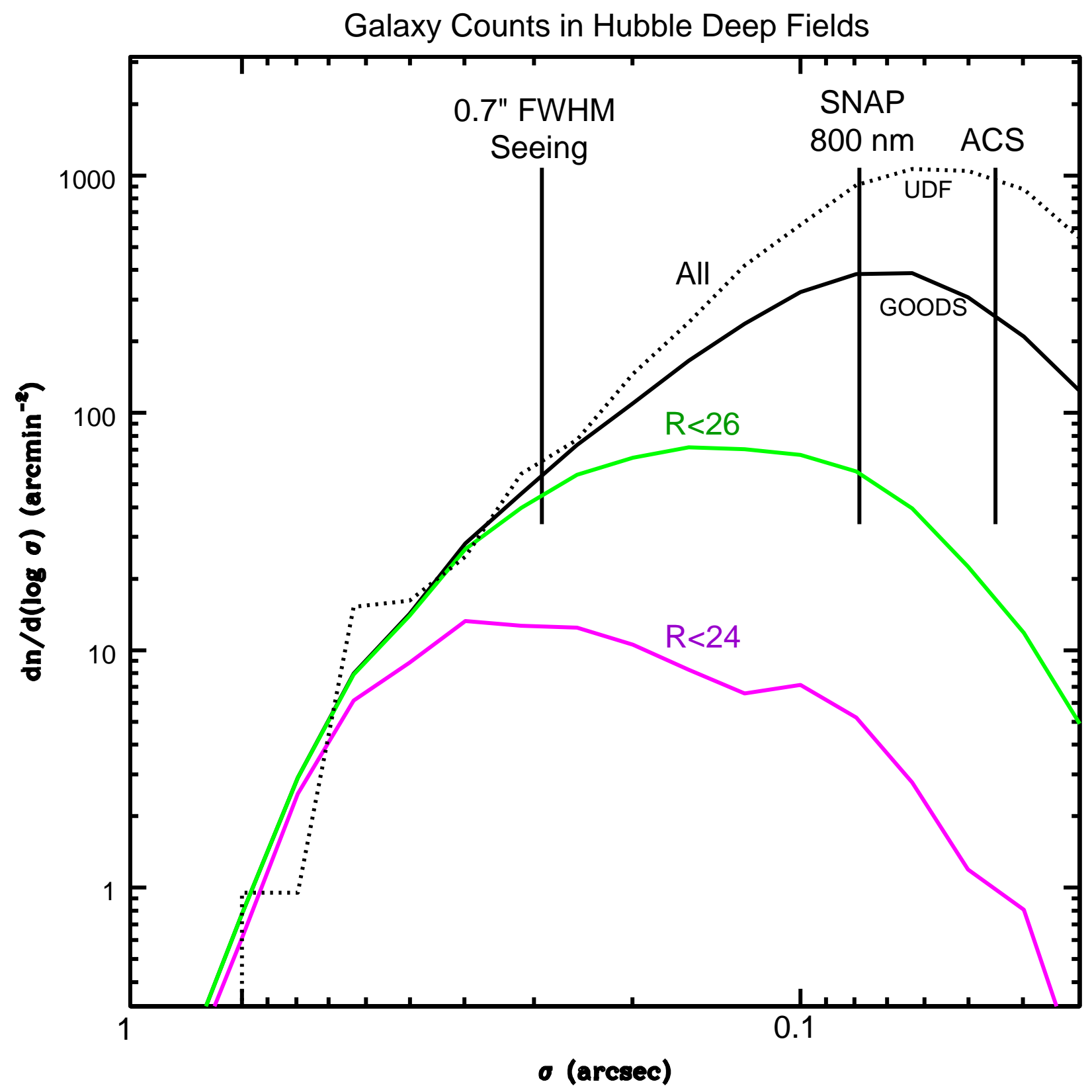

Fig. 2.- The number density of galaxies per square arcminute vs. $\sigma$ as an indicator of galaxy size for a Gaussian profile. These are shown for different limiting magnitudes as indicated. The vertical lines show the achievable number density for instruments with different optical resolution. 

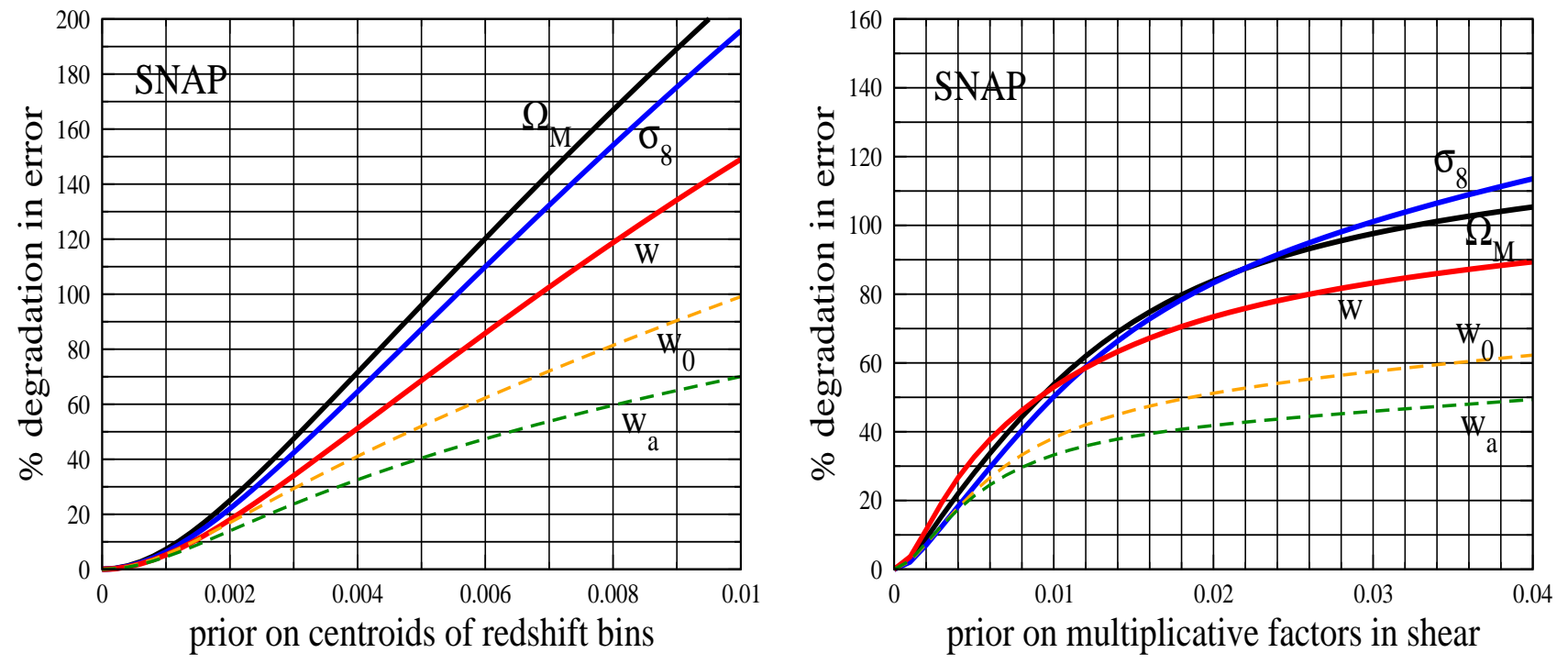

Fig. 3. - Requirements on the redshift biases (left panel) and multiplicative errors (right panel) for the SNAP weak lensing survey; adopted from Huterer et al. (2005). We show the degradations in (marginalized) cosmological parameter errors as a function of the redshift and multiplicative shear bias. Both biases are defined per redshift bin of $\Delta z=0.3$ and are obtained by averaging individual biases of galaxies in that bin. 


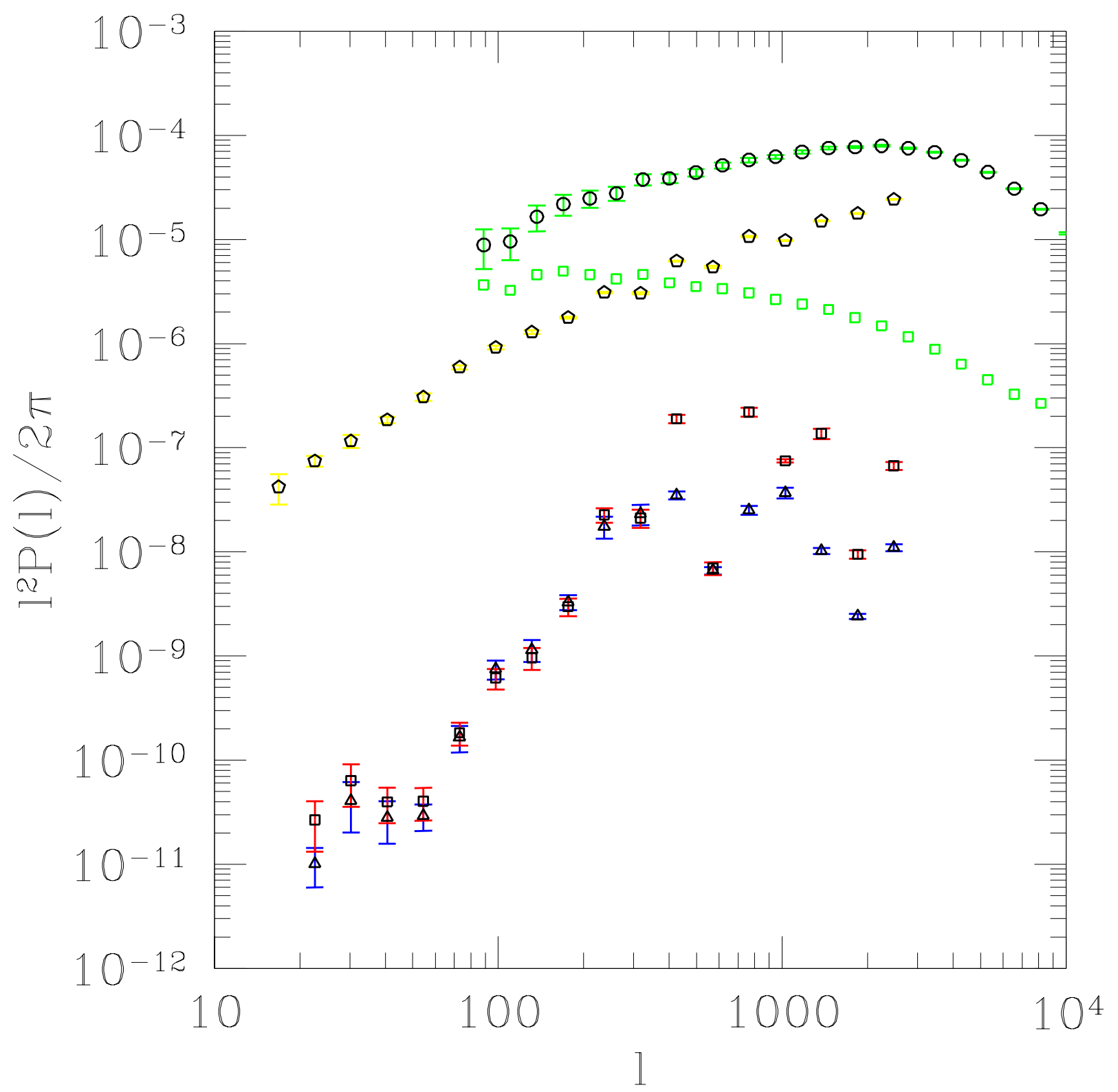

Fig. 4.- Power spectra of the lensing signal and expected PSF anisotropy contamination for the SNAP telescope. Thermal effects and vibration in the structure of the telescope lead to time variation in the PSF anisotropy. This residual power would lead to an additive systematic error in the lensing measurement. The red curve (squares) is the time-varying power due to thermal effects, and has not had the static PSF pattern subtracted off, whereas the blue curve (triangles) is the residual power after the time invariant pattern is subtracted (as it is expected to be measured accurately). The residual power from worst case structural vibrations is shown in yellow (pentagons). The statistical errors for a 1000 square degree survey are shown by the green squares, along with the power spectrum amplitude of the shear signal from simulations (circles). 


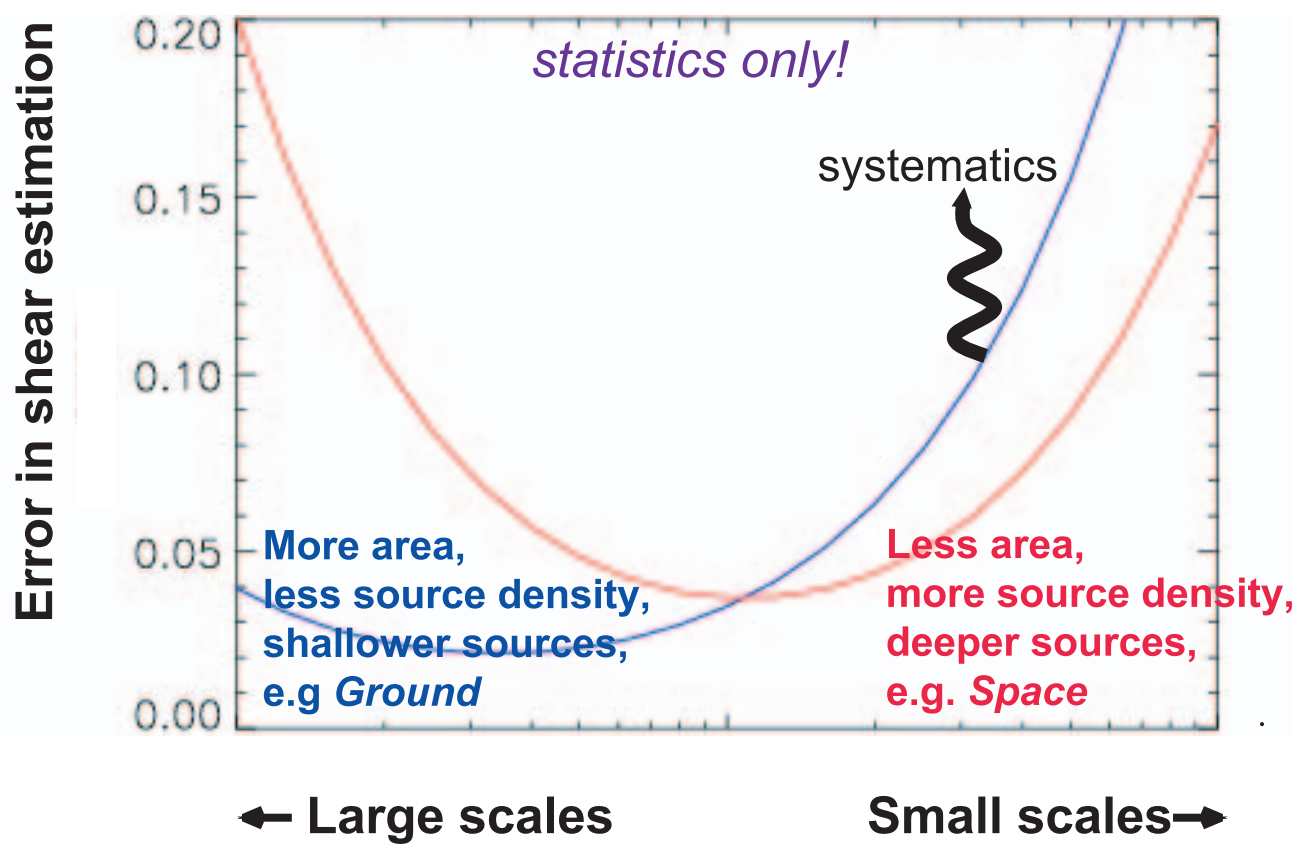

Fig. 5.- A qualitative representation of the error in shear estimation over a range of angular scales from $l=10$ to about $l=10^{4}$. At the largest angular scales (small $l$ ) a very wide survey like LSST will provide smaller errors. At higher $l$ the space-based survey will have smaller errors due to the higher surface density and better shape measurements available. Systematics, which are considerably worse from the ground, will tend to push the blue, ground-based curve up. 


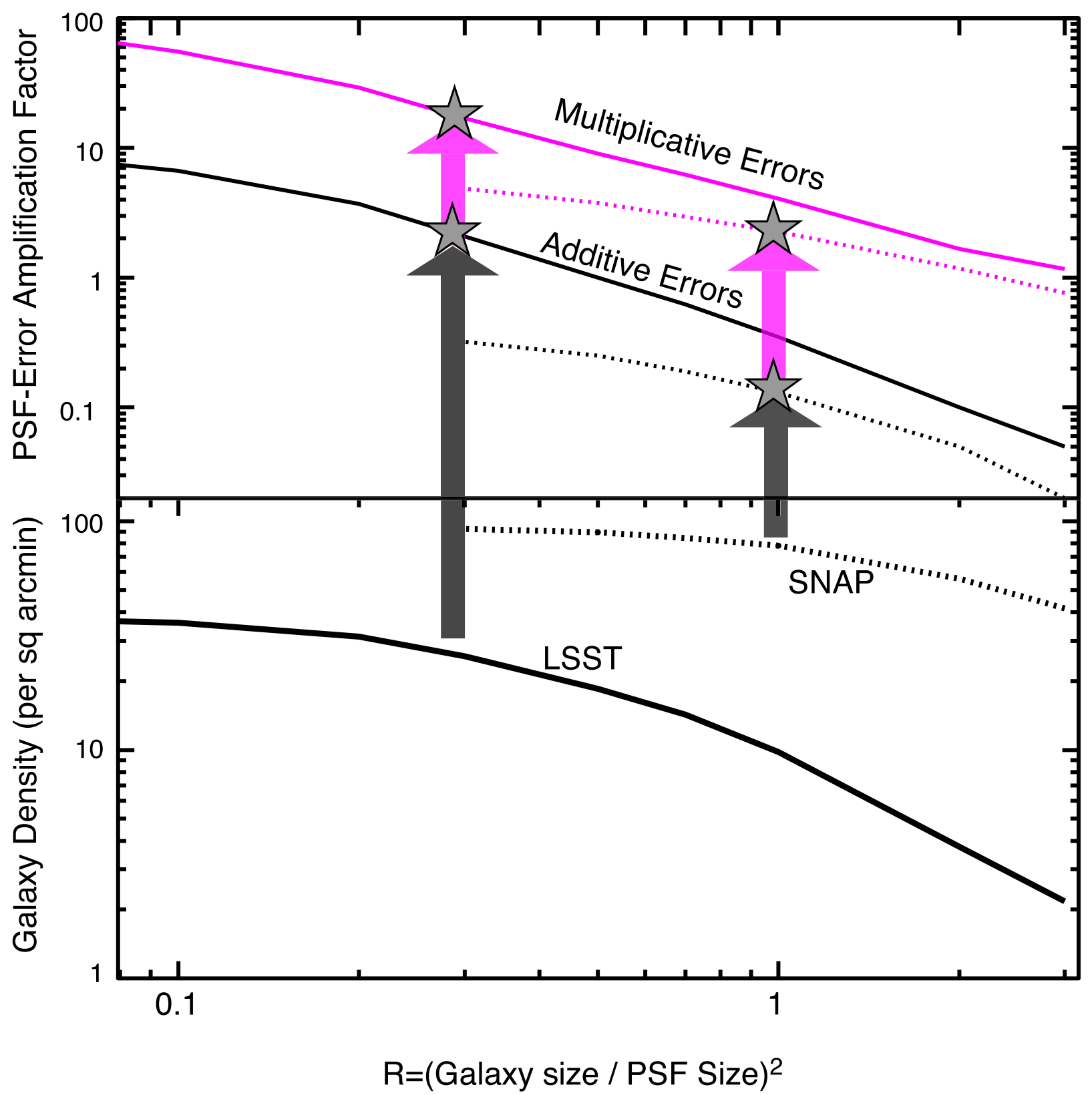

Fig. 6. - Using the Hubble deep fields as a census of the size distribution of galaxies, the bottom panel shows the effective number of measurable galaxies vs. the minimum intrinsic galaxy size (relative to the PSF). For the nominal SNAP survey, most galaxies are resolved, so the curve plateaus below $R=1$. From the ground, most galaxies are marginally or poorly resolved, so the curve continues rising to $R<1$. A plateau is reached for $R \sim 0.3$ because one requires unattainably high $\mathrm{S} / \mathrm{N}$ to measure the shape of more poorly resolved galaxies. This plateau has $3 \mathrm{x}$ fewer galaxies per square arcminute than the nominal SNAP Wide survey. The upper panel plots the factor by which errors in PSF ellipticity (size) determination are amplified into additive (multiplicative) errors in the measured lensing shear power spectrum. As expected, the measured shear becomes very sensitive to PSF errors when one attempts to use poorly-resolved galaxies. We see that a space survey, using galaxies with $R>1$, would have 1-2 orders of magnitude less sensitivity to PSF errors than a ground survey using $R>0.3$ galaxies. 